\title{
HST-FOS spatially resolved spectral classification of compact WR/Of+OB groups in the LMC
}

\author{
Nolan R. Walborn ${ }^{1}$, Laurent Drissen ${ }^{2}$, Joel Wm. Parker ${ }^{3}$, \\ and Abhijit Saha ${ }^{4}$ \\ ${ }^{1}$ Space Telescope Science Institute, Baltimore, Maryland, USA \\ ${ }^{2}$ Université Laval, Ste-Foy, Québec, Canada \\ ${ }^{3}$ SouthWest Research Institute, Boulder, Colorado, USA \\ ${ }^{4}$ Kitt Peak National Observatory, Tucson, Arizona, USA
}

\begin{abstract}
Spectroscopy of the individual stellar components in four compact OB multiple systems of the LMC, previously imaged with the HST-WFPCs, has been performed with the HST-FOS. In every case, the uncontaminated spectra of the WR and/or Of-type components have been observed for the first time. The spectra of the normal OB companions provide information about the initial masses and ages of the peculiar objects. Several additional evolved and peculiar objects within these systems have also been discovered.
\end{abstract}

The Magellanic Clouds present the best available laboratory to investigate the upper stellar mass limit and initial mass function, because of the common distances and low extinction. However, massive stars form preferentially in compact groups, and even in the LMC 1" subtends $50000 \mathrm{AU}$, so that the highest possible spatial resolution is required. The Hubble Space Telescope provides the best current capability. Accordingly, we have imaged several compact WR/Of+OB systems of special interest with the HST-wide Field/Planetary Cameras, and then performed spatially resolved, blue-violet spectroscopy of the brighter individual components in four of them with the HST-Faint object spectrograph. The systems are Breysacher 73 in 30 Doradus B, the West cluster in NGC 2044 (30 Doradus C), HD 32228 in Henize N 11/Lucke-Hodge 9, and Parker 3209 in N 11/LH 10. The direct images of the first two are shown by Walborn et al. (1995a), and the latter two by Walborn et al. (1995b). In every case, the followup FOS observations have revealed the uncontaminated spectra of the WR and/or Of components for the first time. The spectra of the normal OB companions will be used to deduce information about the initial masses and ages of the peculiar objects. However, a number of additional evolved and peculiar objects within these systems have also been discovered. For instance, as well as the WN component, Brey 73 contains peculiar Be and early-O giant members. The former objects may be pre-main-sequence Be stars, while the latter has exactly twice the mass of the current group turnoff, and it is proposed as a massive stellar merger.

As an example of the FOS results, Figure 1 presents the data for P 3209 in LH 10. Walborn \& Parker (1992) showed that the groundbased spectrogram of this object has the characteristic features of type O3 III $\left(\mathrm{f}^{*}\right)$, but combined with strong He I lines, which were assumed to arise in a later-type OB companion. The WFPC image reproduced by Walborn et al. (1995b) indeed reveals that the 


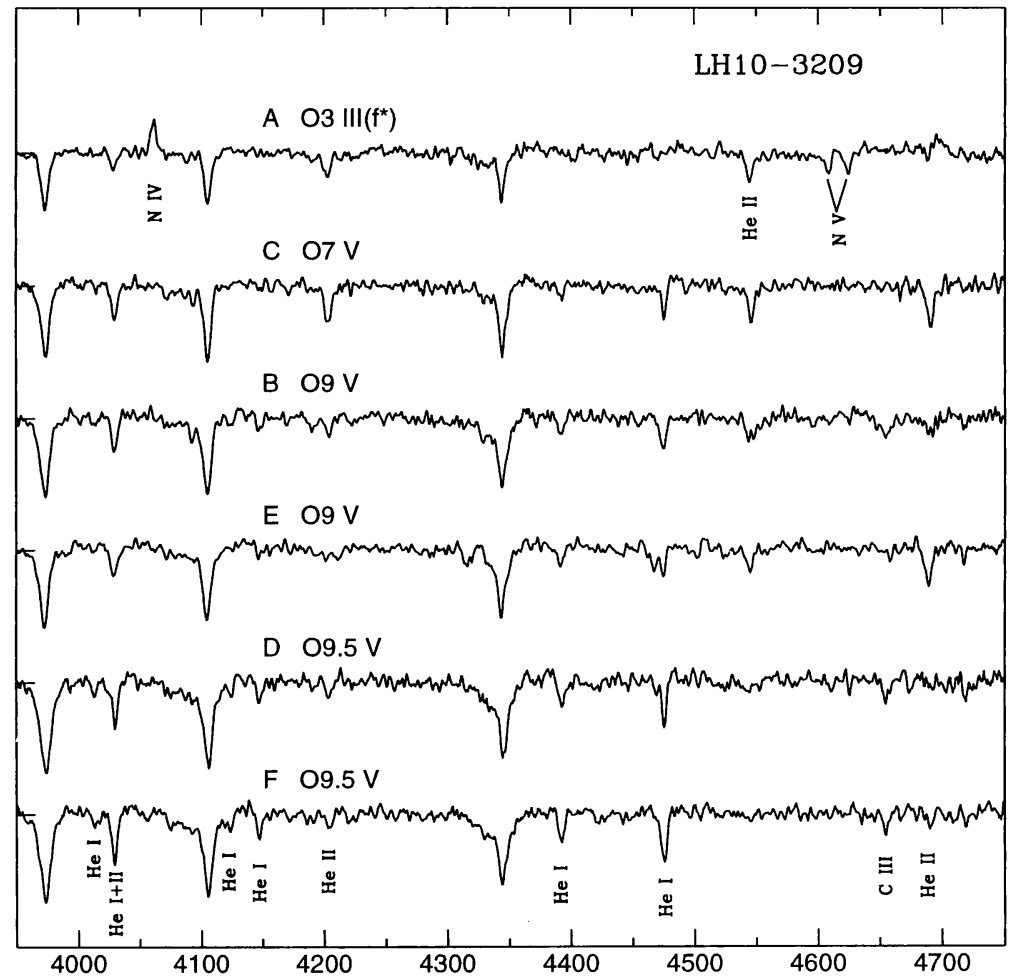

Figure 1. HST-FOS spectra of Parker 3209 objects in LH 10

object is a compact multiple system containing six bright components within a radius of $1^{\prime \prime}$ (while other stars in the association with pure O3 spectra have single images). Finally, the Fos data in the figure complete the story, by establishing that the brightest component is of pure O3 III $\left(\mathrm{f}^{*}\right)$ type, while the He I lines are due entirely to the five mid- and late-O companions. The complete spectroscopic and photometric results from this program will be published shortly in The Astronomical Journal.

Acknowledgments. Support for this work was provided by NASA through grants numbers GO-6032.01-94A and GO-6508.01-95A from STScI, which is operated by AURA, Inc., under NASA contract NAS 5-26555.

\section{References}

Walborn, N.R., MacKenty, J.W., Saha, A., White, R.L., Parker, J.Wm. 1995a, ApJ 439, L47

Walborn, N.R. et al. 1995b, Proceedings of 11th IAP Meeting, p. 61

Walborn, N.R., Parker, J.Wm. 1992, ApJ 399, L87 